\title{
Personalização de Agentes Pedagógicos Animados
}

\author{
Elisa Boff, Universidade de Caxias do Sul - eboff@ucs.br \\ Adriano Oliveski, Universidade de Caxias do Sul - aoliveski@gmail.com
}

Resumo. Com a popularização das tecnologias que dão às instituições de ensino suporte à aprendizagem a distância, novas estratégias pedagógicas vêm sendo utilizadas para facilitar o processo de ensino aprendizagem de seus alunos. Este artigo traz a implementação de uma dessas estratégias pedagógicas: o uso de agentes pedagógicos animados para motivar a participação dos alunos em ambientes virtuais de aprendizagem. Para isso, o trabalho se embasou em ideias e teorias de diversas áreas do conhecimento, como artes, informática e psicologia. Este artigo tem o objetivo de apresentar um sistema para personalização de um agente pedagógico animado a fim de inserir o aluno no processo de criação do seu personagem. Este agente é dotado de personalidade, fazendo com que cada agente seja único, motivando o aluno na utilização e participação em um ambiente virtual de aprendizagem.

Palavras-chave: Agentes Pedagógicos, Ambientes Virtuais de Aprendizagem, Personalidade

\section{Animated Pedagogical Agents'Personalization}

\begin{abstract}
With the popularization of technologies that give educational institutions to support distance learning, new teaching strategies have been used to facilitate the process of teaching and learning of their students. This article presents an implementation of these teaching strategies: the use of animated pedagogical agents to motivate student participation in virtual learning environments. For this, the work is based on ideas and theories from different areas of knowledge such as arts, computer science and psychology. This article aims to present a system for customizing an animated pedagogical agent in order to include the student in the process of creating your character. This agent is endowed with personality, so that each agent is unique, motivating the students to use and participation in a virtual learning environment.
\end{abstract}

Keywords: Pedagogical Agents, Learning Environments, Personality

\section{Introdução}

Com o avanço das tecnologias, em especial das tecnologias de rede e da Internet, e com a facilidade de acesso a essas tecnologias, diversas instituições de ensino tem informatizado seus serviços, realizando desde matrículas online até cursos à distância. Essa popularização das tecnologias fez com que instituições acadêmicas, professores e alunos passassem a usufruir a WEB e meios eletrônicos de comunicação e mediar o processo de ensino-aprendizagem de forma mais colaborativa (Tarouco, 1999). 
Os Ambientes Virtuais de Aprendizagem (AVA) foram desenvolvidos para dar suporte a essas atividades e cursos online. Estes ambientes permitem desenvolver a interação entre os alunos, professores e objetos de estudo, integrar diferentes mídias, linguagens e recursos, a fim de contribuir com o aprendizado do aluno.

Entretanto, essa facilidade de acesso à tecnologia não significa que o aluno comum esteja apto a utilizar estes ambientes eficientemente. Alunos com algum conhecimento prévio sobre tecnologias são capazes de utilizar um AVA sem maiores problemas, porém, esses sistemas são propostos as mais diversas áreas do conhecimento, onde nos deparamos com alunos não familiarizados com tais tecnologias. Estes alunos menos habituados a tecnologias podem se mostrar relutantes na utilização destes ambientes (Barajas e Owen, 2000).

Com o intuito de auxiliar alunos sem os conhecimentos técnicos necessários para utilizar ambientes virtuais, alguns sistemas valem-se de chatterbots. Chatterbots, ou agentes conversacionais, são sistemas que simulam a conversa humana, tendo como objetivo responder perguntas deixando ao usuário a sensação de estar conversando com outra pessoa.

Quando esses agentes possuem o objetivo de auxiliar no conteúdo didático eles passam a ser chamados de Agentes Pedagógicos. Em um AVA, os agentes têm como função identificar dificuldades dos alunos, monitorar o desenvolvimento de tarefas, trazer dicas, entre outros. Segundo Giraffa (1999), Agentes Pedagógicos são aqueles utilizados em sistemas desenvolvidos para fins educacionais, podendo atuar como tutores virtuais, alunos virtuais, ou ainda como companheiros virtuais de aprendizagem, tendo como objetivo auxiliar os alunos no processo de ensino-aprendizagem.

Muitos ambientes se utilizam de Agentes Pedagógicos a fim de orientar os alunos quanto à navegação e realização de tarefas. Porém, durante a criação desses agentes, aspectos importantes são deixados de lado, fazendo com que o usuário fique frustrado com o mesmo, ou seja, personagens perdem sua credibilidade assim que usuário identifica suas limitações. Este artigo apresenta a modelagem de um Agente Pedagógico com características antropomórficas, ou seja, um agente que possua atributos humanos, juntamente com sua ferramenta de personalização, dando ao aluno a possibilidade de criar seu agente de acordo com seu estilo. O agente proposto ainda assumirá uma personalidade correspondente aos modelos de aparência escolhidos pelo usuário no momento de sua criação. Segundo Erickson (1997) o uso de emoções e personalidade em agentes traz um ganho significativo, pois contribui para a ilusão de vida do agente. A simples presença de características humanas faz com que as pessoas apliquem regras sociais aos computadores. Em alguns casos os usuários ficam emocionalmente engajados tentando entender, controlar e prever o funcionamento do agente.

O agente desenvolvido e sua ferramenta de personalização têm o objetivo de auxiliar o aluno em suas atividades acadêmicas dentro do AVA da Universidade de Caxias do Sul.

Este artigo está organizado como segue. A seção 2 apresenta um estudo sobre os Agentes Pedagógicos. A seção 3 traz uma revisão sobre conceitos de Cores e Personalidade, utilizados para modelagem visual e comportamental do agente. A seção 4 detalha o sistema para personalização de Agentes Pedagógicos Animados desenvolvido. Por fim, são apresentadas as considerações finais e as referências 
utilizadas para redação deste artigo.

\section{Agentes Pedagógicos}

Agentes de interface são personagens virtuais utilizados para facilitar a interação entre sistemas computacionais e o usuário. Estes agentes interagem com o usuário através de linguagem natural para, em geral, fornecer ajuda ao usuário na utilização de um sistema computacional. Os Agentes Pedagógicos, por sua vez, são personagens virtuais (animados ou não) que também podem interagir com o usuário em linguagem natural, mas com algum objetivo de aprendizagem, ou de motivar e incentivar a aprendizagem de um aluno.

Mesmo que as interfaces computacionais, na maioria das vezes, não apresentem características antropomórficas, os usuários tendem a aplicar regras sociais aos computadores. Este comportamento vem fazendo com que pesquisadores busquem personificar a interface de seus sistemas, incorporando a elas agentes animados, os quais apresentam comportamentos credíveis, semelhantes aos dos seres humanos (Reategui e Moraes, 2006). Os personagens são capazes de auxiliar os estudantes na realização de tarefas, guiá-los no ambiente, identificar dúvidas, motivar a interação entre estudantes, apresentar dicas, entre outras. Reategui e Moraes (2006) concluem que a utilização de agentes de interface colabora na interação humano-computador, tornando essa interação mais amigável, introduzindo componentes afetivos que auxiliam o aluno em seu processo de aprendizagem.

Kurrupako (Reategui et al., 2006) é um agente de interface sócio-afetivo desenvolvido para auxiliar alunos em um ambiente virtual de ensino de algoritmos. $\mathrm{O}$ agente monitora a interação com o aluno coletando informações para mais tarde fazer recomendações personalizadas de conteúdo. Outra capacidade deste agente é o reconhecimento de outros alunos conectados ao ambiente, que podem se auxiliar em determinados tópicos, motivando a formação de grupos de estudo.

Procurando proporcionar uma interação mais realista entre o chatterbot e o usuário, Moraes e Wilkens (2008) desenvolveram um agente com a capacidade de simular emoções e personalidade que engajem o usuário em uma interação mais atrativa, motivada e útil. $\mathrm{O}$ agente conversacional construído tem como principais características avaliar o diálogo sobre emoções, representar o modelo de emoções Big Five (McCrae e Costa, 1989), e verificar o impacto dos valores emocionais sobre a interface. As tecnologias utilizadas para a criação do chatterbot emocional foram: AIML, para a criação e armazenamento da base de conhecimento do agente; Chatterbean, o interpretador dos arquivos AIML; e XML para indicar propriedades e configurações pré-definidas no Chatterbean. Quanto à interface gráfica, foi utilizado o mesmo padrão de interface que os sistemas de troca de mensagens instantâneas, devido à familiaridade de usuários com estes sistemas. Também foi utilizado o programa $x F a c e$ para criação das várias expressões do avatar.

O agente pedagógico PAT (Jaques e Viccari, 2005) tem como objetivo fornecer um suporte emocional para o aluno, motivando-o e encorajando-o, para que o aluno apresente melhores resultados em seus estudos. O personagem aplica suas táticas afetivas através de mensagens de encorajamento e de comportamentos emotivos. $\mathrm{O}$ agente PAT reconhece as emoções do aluno pelo seu comportamento observável através do modelo OCC (Ortony, Clore \& Colllins, 1988). Esse modelo é baseado na 
abordagem cognitiva das emoções e é possível de ser implementado computacionalmente, já que ele fornece informações de como construir uma interpretação de uma situação do ponto de vista do usuário e para qual emoção esta interpretação nos leva.

\section{Cores e Personalidade}

A origem das diferenças individuais deriva de diversos fatores como, a forma como fomos criados, o ambiente a nossa volta, fatores hereditários, entre outros. Ainda assim a origem dessa conduta humana continua sendo objeto da investigação psicológica.

Comumente utilizamos adjetivos para caracterizar estes traços de personalidade, tais como, sincero, honesto, compreensivo, inteligente, amigável, ambiciosos, pontual, tolerante, irritável, responsável, calmo, artístico, científico, ordeiro, religiosos, falador, excitado, moderado, calado, corajoso, cauteloso, impulsivo, oportunista, radical, pessimista, entre outros. Porém cada pessoa possui muitos desses traços, sendo assim, o que define sua personalidade é a predominância de algum traço e a atenuação de outros.

O modelo dos Cinco Grandes Fatores (McCrae e Costa, 1989) é uma versão criada a partir da Teoria de Traço, e representa um avanço conceitual no campo da personalidade, possibilitando descrever as características humanas básicas de forma sólida e replicável.

$\mathrm{Na}$ área da psicologia, muitos autores trabalharam na construção de uma taxonomia de traços de personalidade, mas foram McCrae e Costa (1989) que descreveram o Modelo dos Cinco Grandes Fatores (Five Factor Model), o qual apresentou cinco dimensões humanas a fim de ter modelos de personalidade consistentes. Os cinco fatores apresentados foram: Neuroticismo/Estabilidade Emocional (Neuroticism), Extroversão (Extraversion), Intelecto/Abertura para Experiência (Openness to Experience/Intellect), Socialização/Afabilidade (Agreeableness) e Escrupulosidade/Consciência (Conscientiousness).

O modelo dos Cinco Grandes Fatores tem suas origens na análise da linguagem utilizada para descrever as pessoas, ela consegue de maneira simples classificar os traços de personalidade reconhecidos tanto por profissionais quanto por leigos. A descrição que segue é um resumo do entendimento do significado dos fatores utilizados neste trabalho.

O fator Extroversão trata da sociabilidade, possuindo em um de seus extremos, características que descrevem o individuo como afetivo, sociável, falante, passional, alegre; no outro extremo as características descrevem o indivíduo como reservado, tímido, quieto, incessível, discreto, etc. Já o fator conhecido como Neuroticismo ou Estabilidade Emocional indica as tendências à ansiedade, depressão, tensão, irracionalidade, emoção, tendência a sentimento de culpa, etc. Seus extremos contrapõem adjetivos como: tranquilo $\mathrm{x}$ tenso, constante $\mathrm{x}$ instável, satisfeito $\mathrm{x}$ autopiedoso, racional x emocional, seguro $\mathrm{x}$ inseguro.

O trabalho descrito neste artigo utilizou o modelo Big Five como uma base para que seja analisada qual personalidade será atribuída ao Agente Pedagógico construído pelo usuário. O agente proposto aborda dois fatores, e seus extremos, que se mostram mais relevantes para serem aplicados aos agentes pedagógicos, o fator Extroversão e o 


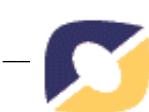

fator Neuroticismo.

Ao escolher uma cor quando tristes, entediados ou desanimados, algumas pessoas optam pelo cinza, para outras essa cor representa seriedade, sabedoria. Quando alegres certas pessoas tendem a escolher o vermelho, enquanto outros veem esta cor representando vulgaridade, barbarismo. Apesar de sempre existir algo de relativo na escolha de uma cor, é verificada a existência de um peso psicológico nessa escolha.

É no córtex do cérebro humano que são realizadas todas as interpretações do meio ambiente, inclusive as cores. A distinção denominação, identificação e qualquer outra reação estética às cores é resultado do desenvolvimento e educação do indivíduo.

As cores constituem estímulos psicológicos para a sensibilidade humana, exercendo influências nas pessoas, fazendo-as negar ou afirmar, gostar ou não de algo, se abster ou agir. Todos nós possuímos ligações afetivas por algumas cores ou mesmo uma gama delas, e esse comportamento de fidelidade faz parte de nosso cotidiano por muito tempo, sem percebermos isso. Baseando-se nestas afirmações, psicólogos estão em comum acordo quanto a atribuições de significados às cores, comuns a indivíduos de nossa cultura.

Analisando os significados psicológicos das cores podemos perceber que os estímulos provindos das cores podem ser empregados como diagnóstico para os tipos de personalidade. Jung (1947) propõe a divisão dos grupos psicológicos conforme a predominância dos fatores de introversão e extroversão, atribuindo cores como, o azul aos indivíduos racionais, verde aos sensitivos, aos sentimentais o vermelho e o amarelo aos sujeitos intuitivos.

Podemos relacionar também a preferência dos indivíduos entre cores quentes e frias. Pessoas inclinadas às cores quentes são caracterizadas por possuírem uma relação muito íntima com o seu ambiente, são receptivos, possuem calor humano, sugestionamse facilmente, são afetivos e abertos à influencias exteriores. Os sujeitos que tendem a escolher cores frias dificilmente se adaptam espontaneamente ao ambiente, são frios e reservados.

Tendo como fato comprovado que a estrutura da personalidade pode ser deduzida a partir do conhecimento de estímulos e comportamento de determinado indivíduo, e sabendo que a cor tem importante influência nesses estímulos, este trabalho utilizou a cor como um dos itens de análise da personalidade a ser agregada aos Agentes Pedagógicos.

\section{Sistema para Personalização de Agentes Pedagógicos Animados}

A implementação do sistema foi dividida em quatro etapas: $O$ design dos personagens, o desenvolvimento da ferramenta de criação e personalização dos agentes, a análise da personalidade e a geração das animações.

A Figura 1 apresenta a interface principal e sua relação com a base de conhecimento do Agente Pedagógico. A pergunta enviada pelo usuário é capturada e analisada na base de perguntas e respostas AIML, essa base determina qual a resposta e reação serão retornadas pelo agente. Essas informações são passadas à base de personagens, onde estão os arquivos XML de cada usuário contendo as informações de aparência e personalidade de seu agente pedagógico. Nessa etapa é feita a união da animação determinada pela base AIML com a aparência escolhida pelo aluno. Por fim, a 


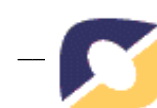

animação é apresentada ao aluno juntamente com a resposta correspondente a sua pergunta. Para este protótipo, a base AIML foi criada com informações referentes a ajuda na utilização das ferramentas e navegação no Ambiente Virtual de Aprendizagem.

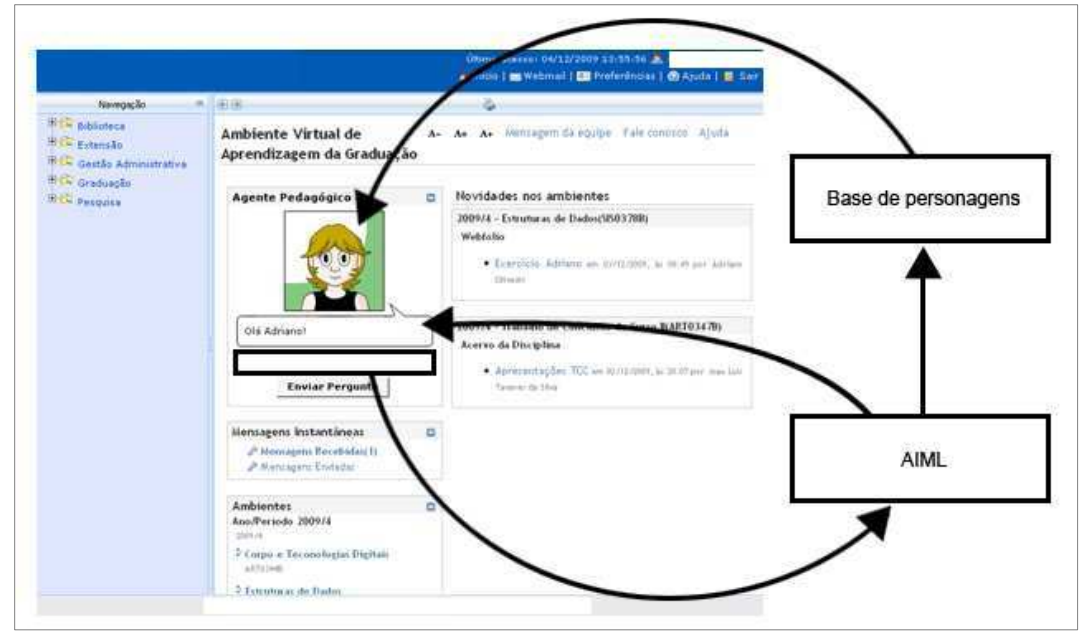

Figura 1 - Arquitetura do Sistema Proposto

Muito utilizado em jornais e na televisão, o estilo escolhido para o desenho dos personagens foi o cartoon. A relação entre cartoon e humor é muito estreita. Partindo deste princípio, este estilo foi escolhido por ser mais carismático, lembrando que a utilização do humor também pode ser um fator motivador na interação com o agente.

Entre modelos de cabelos e roupas, masculinos e femininos, foram criados 50 modelos de aparência. Juntando isso às variações de fundos é possível a criação de mais de 3000 personagens diferentes (Figura 2). Alguns modelos variam apenas em sua cor. Essa variação de cores é utilizada para definir a personalidade do agente seguindo a teoria das cores e traços de personalidade.

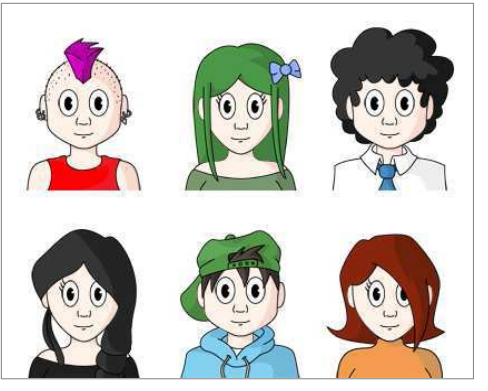

Figura 2 - Alguns dos diferentes modelos de aparência possíveis para os agentes

O principal objetivo da ferramenta proposta neste artigo é o de que, com a possibilidade de criar seu próprio Agente Pedagógico, o aluno sinta-se motivado a utilizar este agente com maior frequência e credibilidade, possibilitando que suas experiências de aprendizagem sejam melhoradas com a ajuda do agente. A motivação faz com que os alunos explorem o ambiente de aprendizagem com mais segurança, ampliando seu contato com o objeto do conhecimento e possibilitando novas e variadas experiências. A ferramenta permite a criação de 3024 aparências diferentes para o Agente Pedagógico, possibilitando que cada aluno possua um agente único, e com sua própria personalidade. 
A interface da ferramenta de personalização (Figura 3) foi pensada para simples, tornando intuitiva a criação dos personagens. O layout, construído com a tecnologia Photoshop CS3, seguiu o padrão de cores de interface do portal acadêmico UCSVirtual, local onde está integrada a ferramenta. Para indicar qual parte do modelo seria alterada foram utilizados ícones no lugar de palavras (cabelo, roupa, fundo ou sexo). Foi optado pela forma gráfica de comunicação, onde os elementos destacados indicam o conteúdo do botão.

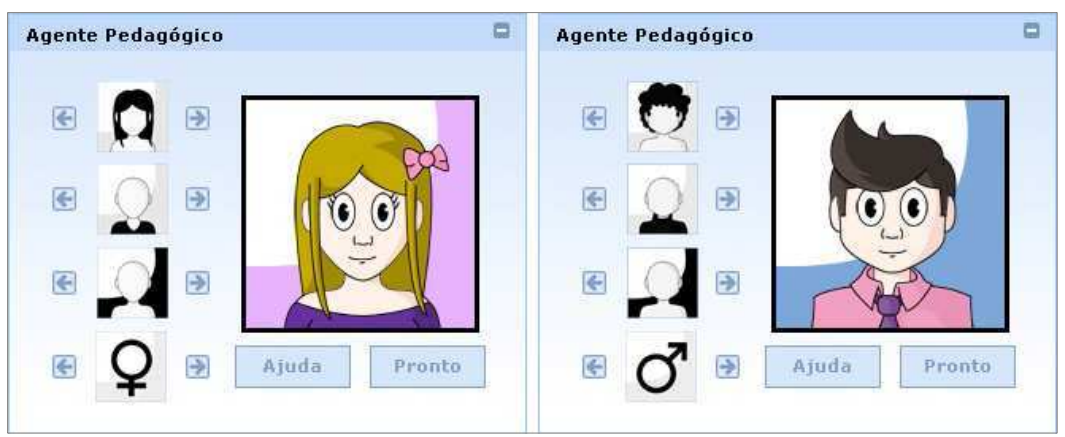

Figura 3 - Interface da ferramenta de personalização dos agentes

Definidos os traços de personalidade a serem trabalhados, foram analisados os modelos descritos na seção 3. Estudando as teorias de aparência e personalidade, as combinações de aparência foram estereotipadas em grupos sociais (Figura 4). Quatro dessas combinações foram destacadas e divididas de acordo com os tipos de personalidade descritas na Tabela 1.

Tabela 1 - Quatro tipos de personalidade atribuída aos agentes pedagógicos

\begin{tabular}{c|l|l}
\hline Personalidade & \multicolumn{1}{|c}{ Características } & \multicolumn{1}{c}{ Grupo social associado } \\
\hline 1 & Reservado, tímido, quieto e discreto. & Geeks \\
\hline 2 & Tranquilo, constante, racional e seguro. & Conservadores \\
\hline 3 & Tenso, instável, emocional e inseguro. & Emo \\
\hline 4 & Afetivo, associável, falante e alegre. & Regueiros \\
\hline
\end{tabular}

O grupo social intitulado Geeks descreve membros com dificuldades de integração social, tímidos e solitários, encaixando-os, portanto, no tipo de personalidade 1. Ao tipo de personalidade 2, foram associados os conjuntos de modelos mais conservadores, que não fogem dos parâmetros impostos pela sociedade, esses modelos representam um individuo constante, sério, etc. $\mathrm{O}$ grupo social rotulado como Emo corresponde ao tipo de personalidade 3 , pois seus membros são conhecidos por serem sentimentais, emotivos. Ao tipo de personalidade 4, foram associados os modelos referentes ao grupo social regueiros, por seus membros possuírem características como sociáveis e alegres.

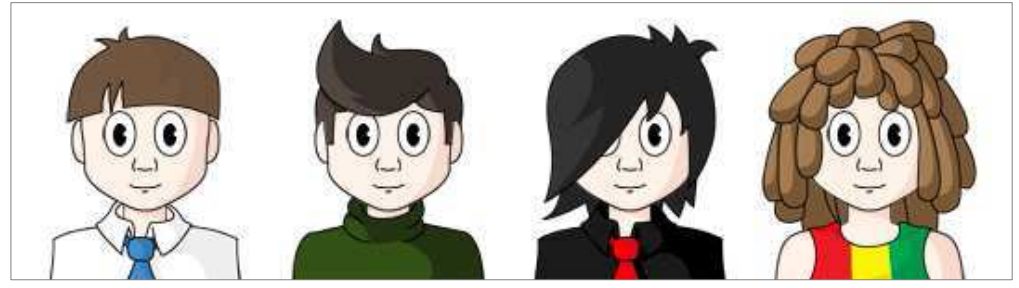

V. $10 \mathrm{~N}^{\mathrm{o}}$ 3, dezembro, 2012 
Figura 4 - Modelos representando grupos sociais

Baseado nos modelos de aparência descritos, foi criado um sistema de pontuação para definir o tipo de personalidade a ser atribuído ao agente. Cada modelo de cabelo ou roupa possui um valor, e o resultado de sua soma corresponde a um tipo de personalidade. A aparência (cabelo, vestuário) está relacionada a personalidade, conforme apresentado na Figura 2. A Tabela 2 apresenta os valores referentes a cada tipo de personalidade, sendo SM o resultado da soma dos modelos de aparência.

Tabela 2: Valores referentes a cada tipo de personalidade

\begin{tabular}{c|c|c|c}
\hline Personalidade 1 & Personalidade 2 & Personalidade 3 & Personalidade 4 \\
\hline $\mathrm{SM}<6$ & $5<\mathrm{SM}<11$ & $10<\mathrm{SM}<16$ & $15<\mathrm{SM}$ \\
\hline
\end{tabular}

Tendo como base estes modelos, iniciaram-se as atribuições de valores aos demais modelos de aparência criados. Com o auxílio dos estudos sobre cores e traços de personalidade, consideramos a cor utilizada nos modelos restantes como um fator de pontuação, ou seja, modelos iguais, mas com cores diferentes recebem valores diferentes, de acordo com suas cores. Seguindo os estudos realizados, as cores foram associadas a cada tipo de personalidade definido na Tabela 1. As cores frias estão relacionadas aos tipos de personalidade 1 e 2 , diminuindo a pontuação dos modelos que utilizam essas cores, enquanto as cores quentes correspondem com os tipos de personalidade 3 e 4, aumentando a pontuação dos modelos que apresentam essas cores.

Outro fator de análise da personalidade é o fundo que o aluno determinar para seu agente. Cada fundo também recebe uma pontuação que é somada aos valores dos modelos de cabelo e roupa. $\mathrm{O}$ valor do fundo segue a mesma metodologia: cores frias, valores baixos e cores quentes, valores maiores. As cores monocromáticas (preto e branco) recebem valores nulos.

A personalidade atribuída ao agente não se faz relevante se esta não for percebida pelo aluno. Pensando nisso animações foram criadas para simular as reações dos agentes a determinados estímulos de acordo com sua personalidade.

No AVA, o Agente Pedagógico se comporta de acordo com a interação com o aluno. Quando o usuário faz uma pergunta rude ao agente é realizada a chamada da animação que apresenta a reação àquela pergunta (Figura 5).

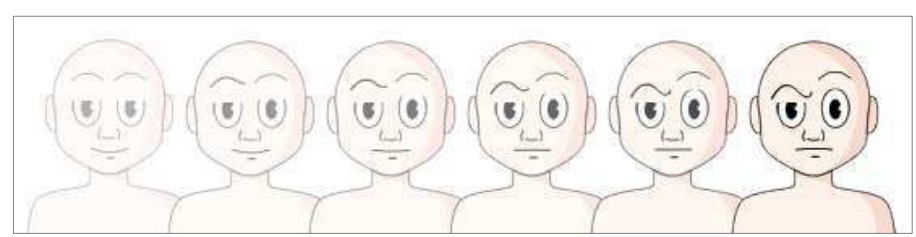

Figura 5 - Reação do agente de personalidade tipo 2 a uma pergunta rude

Após isso o estado do agente não volta ao normal, ele permanece em seu estado de perturbação, surpresa ou braveza, de acordo com sua personalidade. $\mathrm{O}$ mesmo acontece quando o aluno realiza uma pergunta em que o agente pedagógico não é capaz de responder. O estado volta ao normal, neutro, quando o usuário realiza uma pergunta em que o agente é capaz de ajudá-lo, com uma expressão de tranquilidade e satisfação (Figura 6). Essas mudanças ou permanências de estados ocorrem para fortalecer as características de antropomorfismos, procurando atribuir credibilidade ao agente 


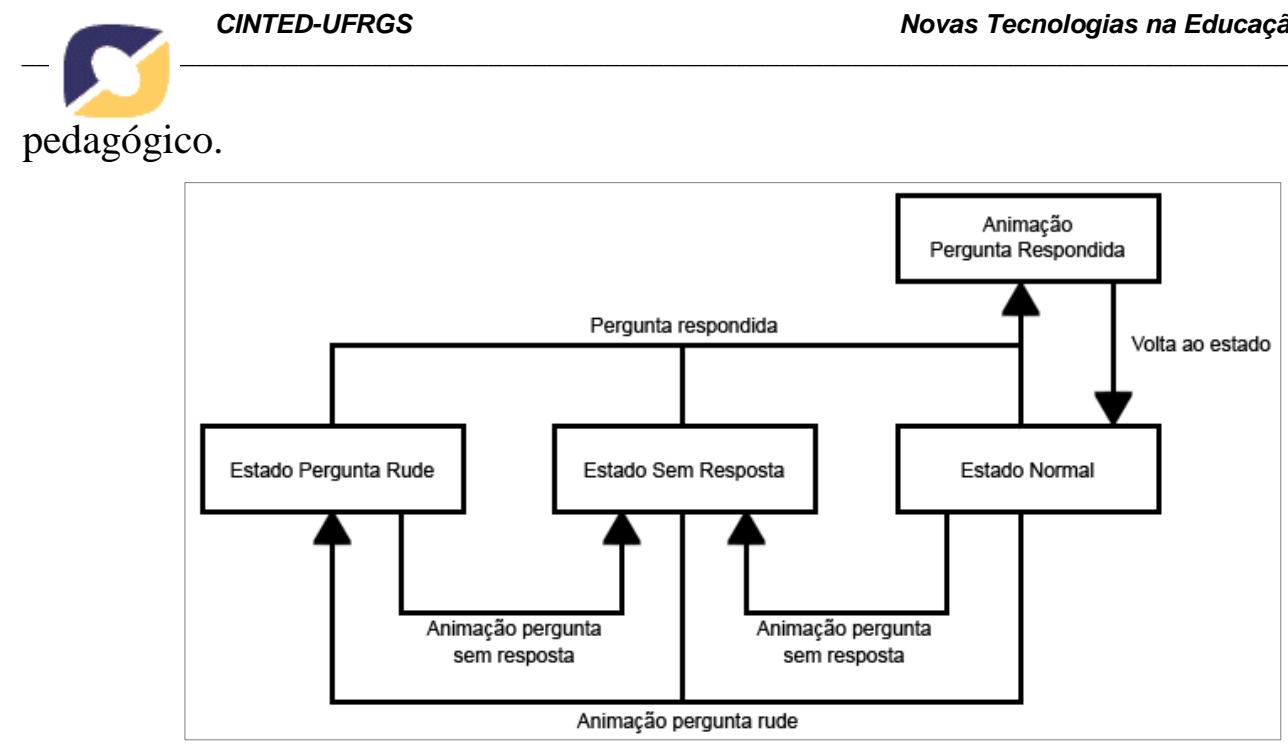

Figura 6 - Estados do Agente Pedagógico

A Figura 7 apresenta uma animação com a troca de estado de uma resposta mal sucedida a um estado de resposta bem sucedida.

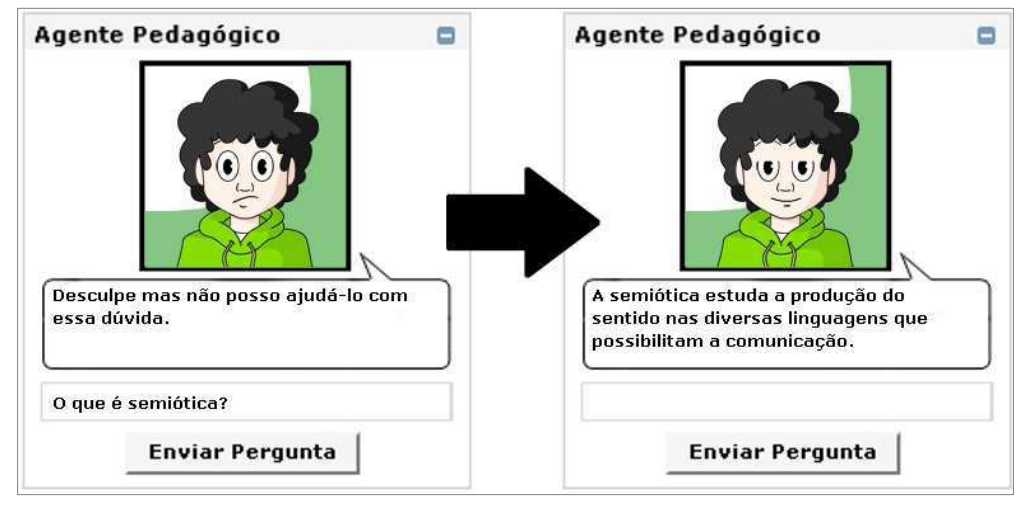

Figura 7 - Animação que demonstra a reação em uma ajuda bem sucedida

\section{Considerações Finais}

Este artigo apresenta o processo de desenvolvimento de uma ferramenta que permite ao aluno, usuário de um ambiente virtual de aprendizagem, criar seu próprio agente pedagógico, gerando empatia com o mesmo, e consequentemente, introduzindo um componente afetivo que pode facilitar o processo de aprendizagem.

No artigo foi discutida a importância de um Agente Pedagógico, bem como os aspectos importantes na criação do mesmo, procurando tornar o agente credível, com características antropomórficas, evitando que o usuário fique frustrado com o mesmo.

A ferramenta projetada e desenvolvida possibilita de forma simples e intuitiva a criação de mais de 3000 combinações diferentes de aparência. A personalidade é outro ponto a ser destacado, com reações diferentes a cada situação, o agente torna-se interessante para o aluno, motivando-o a ter um uso contínuo do mesmo, permitindo dessa forma que o agente lhe apoie em seus estudos.

Como trabalhos futuros, podemos indicar a criação de outros modelos de aparência, associados a outros grupos sociais, procurando aumentar as opções de estilo 
do aluno ao criar seu agente; a criação de novas animações de reações do agente, referentes a outros estímulos recebidos durante a interação com o aluno e a utilização de técnicas de inteligência artificial a fim de identificar, na base AIML, o tipo de pergunta feita por este aluno. As perguntas ou comentários podem ser do tipo pergunta, comentário trivial, comentário rude, reclamação, entre outros. Uma validação deste sistema foi detalhada em Boff (2012).

\section{Referências}

BARAJAS, M., OWEN, M. (2000). Implementing Virtual Learning Environments: Looking for Holistic Approach. Educational Technology \& Society 3(3) 2000. Disponível em: <http://ifets.fit.fraunhofer.de/periodical/vol_3_2000/barajas.html> Acesso em: junho, 2009.

BOFF, E. and REATEGUI, E. B. Mining Social-Affective Data to Recommend Student Tutors. Advances in Artificial Intelligence - IBERAMIA 2012. Lecture Notes in Computer Science, Volume 7637, 2012, pp 672-681. 2012.

ERICKSON, T. Design Agent As If People Mattered. In: Software Agents. Menlo Park, California: AAAI Press, 1997.

FARINA, Modesto. Psicodinâmica das Cores em Comunicação. 2.ed. São Paulo: Edgar Blücher, 1986, pp. 223.

GIRAFFA, L. M. M. Uma arquitetura de tutor utilizando estadas mentas, 1999. Tese (Doutorado em Ciências da Computação) - Instituto de Informática, UFRGS, Porto Alegre. 1999.

GOLDBERG, L. The Development of Makers for de Big-Five Factor Structure. Psychological Assessment, [S.1.], v. 4, n. 1, p. 26-41, 1992.

JAQUES, Patrícia Augustin; VICCARI, R. M.. PAT: Um Agente Pedagógico Animado para Interagir Afetivamente com o Aluno. RENOTE, v. 3, p. 1-19, 2005.

JUNG, C. G. Tipos Psicológicos. Buenos Aires: Sudamérica, 1947.

MCCRAE, R. R.; COSTA, P. T. More reasons to adopt the Five-Factor Model. American Psychologist, [S.1.], v. 44, p. 451-452. 1989.

MORAES, M. C.; WILKENS, Rodrigo. Um Agente Conversacional Emocional. Hífen (Uruguaiana), v. 32, p. 107-114, 2008.

ORTONY A., CLORE G., COLLINS A. The cognitive structure of emotions. Cambridge, UK: Cambridge University Press, 1988.

REATEGUI, Eliseo Berni; BOFF, Elisa; CERON, Rafael Fernando; VICARI, Rosa Maria. Kurrupako: Um Agente Animado Sócio-Afetivo para Ambientes de Aprendizagem. RENOTE, v. 4, p. a27_21184, 2006.

REATEGUI, Eliseo Berni; MORAES, Marcia. Agentes Pedagógicos Animados. RENOTE, v. 4, p. 1-10, 2006.

TAROUCO, Liane. O Processo de Avaliação na Educação a Distância, 1999. Disponível

em: <http://www.pgie.ufrgs.br/webfolioead/biblioteca/artigo6/artigo6.html> Acesso em: 
novembro, 2009. 